\title{
Ulinastatin attenuates diabetes-induced cardiac dysfunction by the inhibition of inflammation and apoptosis
}

\author{
WEN-KE WANG ${ }^{1 *}$, QING-HUA LU ${ }^{1 *}$, XIN WANG $^{1}$, BEN WANG $^{2}$, JUAN WANG $^{1}$, \\ HUI-PING GONG ${ }^{1}$, LIN WANG ${ }^{3}, \mathrm{HAO} \mathrm{LI}^{1}$ and YI-MENG DU ${ }^{1}$ \\ ${ }^{1}$ Department of Cardiology, The Second Hospital of Shandong University, Jinan, Shandong 250033; ${ }^{2}$ Department of \\ General Surgery, Qilu Hospital of Shandong University, Jinan, Shandong 250012; ${ }^{3}$ Department of Healthcare, \\ The Second Hospital of Shandong University, Jinan, Shandong 250033, P.R. China
}

Received September 28, 2016; Accepted May 19, 2017

DOI: $10.3892 /$ etm.2017.4824

\begin{abstract}
Ulinastatin exhibits anti-inflammatory activity and protects the heart from ischemia/reperfusion injury. However, whether ulinastatin has a protective effect in diabetic cardiomyopathy is yet to be elucidated. The aim of the present study was to investigate the protective effects of ulinastatin against diabetic cardiomyopathy and its underlying mechanisms. A C57/BL6J mice model of diabetic cardiomyopathy was used and mice were randomly assigned to three groups: Control group, diabetes mellitus (DM) group and DM + ulinastatin treatment group. Cardiac function was assessed using echocardiography and the level of inflammatory cytokine high mobility group box 1 (HMGB1) expression was measured using histopathological examination and reverse transcription-quantitative polymerase chain reaction. The levels of tumor necrosis factor (TNF)- $\alpha$ and interleukin (IL)-6 were measured using western blotting and ELISA. The apoptosis rate in the myocardium was assessed by TUNEL assay. Caspase-3 activation, expression of B-cell lymphoma 2 (Bcl-2) and Bcl-2 associated x (Bax) were measured using western blotting, as was the activity of the mitogen activated protein kinase (MAPK) signaling pathway. The results indicated that ulinastatin significantly improved cardiac function in mice with DM. Ulinastatin treatment significantly downregulated HMGB1, TNF- $\alpha$ and IL-6 expression $(\mathrm{P}<0.05)$ and significantly reduced the percentage of apoptotic cardiomyocytes $(\mathrm{P}<0.05)$ via reduction of caspase-3 activation and the ratio of $\mathrm{Bax} / \mathrm{Bcl}-2$ in diabetic hearts $(\mathrm{P}<0.05)$. In addition, ulinastatin attenuated the activation of the MAPK signaling pathway. In conclusion, ulinastatin had
\end{abstract}

Correspondence to: Dr Yi-Meng Du, Department of Cardiology, The Second Hospital of Shandong University, 247 Bei Yuan Road, Jinan, Shandong 250033, P.R. China

E-mail: yimengd@163.com

*Contributed equally

Key words: ulinastatin, diabetic cardiomyopathy, inflammation, apoptosis a protective effect against DM-induced cardiac dysfunction in a mouse model. This protective effect may be associated with the anti-inflammatory and anti-apoptotic abilities of ulinastatin via the MAPK signaling pathway.

\section{Introduction}

Diabetic cardiomyopathy (DCM) is responsible for increased morbidity and mortality in patients with diabetes mellitus (DM) (1). The pathophysiology of DCM includes myocardial apoptosis, hypertrophy and cardiac fibrosis (2). Hyperglycemia is regarded as an inflammatory condition with the increased expression of pro-inflammatory cytokines in the myocardium (3). Cardiomyocyte apoptosis is an important process induced by inflammation and oxidative stress in the diabetic heart, which eventually leads to cardiac dysfunction (4). Therefore, inhibition of cardiac inflammation and apoptosis is important in the prevention and treatment of DCM (5).

Ulinastatin is an endogenous inhibitor of proteases, which is located in the urine and blood (6). It is reported that ulinastatin may inhibit various serine proteases, including trypsin, chymotrypsin, neutrophil elastase and plasmin (7). On the basis of the multivalent nature of protease inhibition, ulinastatin has been used as a myocardial protective agent $(8,9)$. Ulinastatin has been reported to improve cardiac dysfunction, reduce myocardial infarct size and decrease serum levels of inflammatory cytokines during hemorrhagic shock and ischemia-reperfusion (I/R) injury in the heart $(10,11)$. Previous investigations have demonstrated that the protective effect of ulinastatin is attributed to its anti-inflammatory, anti-oxidative stress and anti-apoptotic properties $(12,13)$. However, the therapeutic effects of ulinastatin in mice with DM remain unclear.

Hyperglycemia-induced inflammation serves an important role in the pathogenesis of DCM (14). As described in our previous study, high mobility group box 1 (HMGB1) is a mediator of inflammation in the pathophysiology of DCM (15). HMGB1 is translocated from the nucleus to the cytoplasm and secreted into the extracellular matrix in the diabetic myocardium (15). Furthermore, cardiomyocytes and cardiac fibroblasts are the principal sources of released HMGB1 (15). It has been reported that ulinastatin is able to reduce the expression of HMGB1 in hepatic I/R injury (16). 
Based on these reports, it may be speculated that ulinastatin is able to inhibit HMGB1 expression, thereby reducing the inflammatory response in DCM. Tumor necrosis factor (TNF)- $\alpha$, interleukin (IL)-6 and IL-10 are cytokines associated with the inflammatory response (17). During the inflammatory response, TNF- $\alpha$ is initially released and regulates IL-6 and IL-8 levels (18). A previous study demonstrated that the protective effects of ulinastatin may be attributed to a number of anti-inflammatory mechanisms (19). Furthermore, ulinastatin was reported to inhibit TNF- $\alpha$ and IL- 6 and increase IL-10 levels in sepsis (20). A previous study provided evidence that ulinastatin inhibits lipopolysaccharide-induced TNF- $\alpha$ upregulation and subsequent IL- $1 \beta$ and IL- 6 induction by macrophages, possibly via the mitogen-activated protein kinase (MAPK) signaling pathway and utilizing extracellular signal-related kinase 1/2, c-Jun N-terminal kinase (JNK) and p38 in vitro (21). Ulinastatin was demonstrated to significantly reduce the phosphorylation of $\mathrm{p} 38$ and $\mathrm{JNK}$ in I/R myocardium injury (22), and DM-induced cardiac dysfunction is reportedly associated with activation of MAPKs, including p38 and JNK (23). The aim of the present study was to investigate whether ulinastatin protects against DCM and to explore the underlying mechanisms responsible.

\section{Materials and methods}

Establishment of type 1 DM model in mice. The study protocol was approved by the Institutional Ethics Committee of Shandong University (Jinan, China). A total of 24 C57/BL6J male mice (25-30 g, 6-8 weeks old) were purchased from Beijing Weitong Lihua Experimental Animal Technology Co., Ltd. (Beijing, China). All mice were housed under standard conditions (12-h light/dark cycle, $22^{\circ} \mathrm{C}$ room temperature, $50 \%$ humidity) and given a normal diet with free access to water. Mice were randomly assigned to two groups: Control $(n=8)$ and DM groups $(n=16)$. Diabetes was induced in mice in the DM group via the injection of streptozotocin (STZ; Sigma-Aldrich; Merck KGaA, Darmstadt, Germany) dissolved in $0.1 \mathrm{ml}$ citrate buffer ( $\mathrm{pH} 4.5$ ) intraperitoneally at a dose of $50 \mathrm{mg} / \mathrm{kg}$ per mouse for 5 consecutive days. The equivalent volume of citrate buffer was administered to mice in the control group. Blood glucose was measured using a glucometer (Accu-Chek Advantage; Roche Diagnostics, Indianapolis, IN, USA). At 1 week after the course of STZ administration, mice with random blood glucose ( $\mathrm{RBG}$ ) levels $\geq 16.7 \mathrm{mmol} / \mathrm{l}$ were considered to be successful diabetic mouse models. Following the induction of diabetes for 8 weeks, mice in the DM group were divided into two groups: DM group $(n=8)$ and $\mathrm{DM}+$ ulinastatin group $(\mathrm{n}=8)$. Body weight, plasma glucose levels, heart rate and systolic blood pressure were measured at 0,8 , and 12 weeks after the STZ or saline treatment.

Ulinastatin treatment. Ulinastatin was obtained from Tianpu Biochemical Pharmaceutical Co., Ltd. (Guangzhou, China). Ulinastatin was administered intraperitoneally at a dose of $100,000 \mathrm{U} / \mathrm{kg}$ daily to mice in the DM + ulinastatin group for 4 weeks, whereas the DM group received $0.9 \%$ saline alone. The dosage of $100,000 \mathrm{U} / \mathrm{kg}$ ulinastatin was selected as this dose has been reported to have a protective effect on mouse organs $(24,25)$. At 12 weeks, mice were sacrificed.
All experiments conformed to National Institutes of Health Guidelines for the Care and Use of Laboratory Animals.

Cardiac function measurements. Echocardiography was performed with the aid of the VisualSonics Vevo 770 imaging system (VisualSonics, Inc., Toronto, ON, Canada) on all animals at baseline and at 8 and 12 weeks after the injection of STZ. Longitudinal and transverse parasternal and 4-chamber apical views were obtained. The M-mode and 2-dimensional imaging views were examined. Left ventricular internal dimensions in systole (LVIDs) and left ventricular ejection fraction (LVEF) were estimated. Pulsed-wave Doppler echocardiography was used to measure early (E) and late (A) blood flow velocities through the mitral valve, and the E/A ratio was calculated.

Myocardial histology. The heart tissues were fixed in $4 \%$ formalin and embedded in paraffin. Tissues were sectioned into $4-\mu \mathrm{m}$ slices for histological studies. All sections were boiled in sodium citrate buffer solution $\left(\mathrm{pH} \mathrm{6.0)}\right.$ at $95^{\circ} \mathrm{C}$ for $15 \mathrm{~min}$, incubated in $30 \% \mathrm{H}_{2} \mathrm{O}_{2}$ for $10 \mathrm{~min}$ at room temperature and subsequently incubated with $5 \%$ fetal bovine serum (Invitrogen; Thermo Fisher Scientific, Inc., Waltham, MA, USA) for $30 \mathrm{~min}$ at room temperature. Sections were next incubated with primary antibodies against rabbit HMGB1 (1:1,000 dilution; ab18256; Abcam, Cambridge, MA, USA) overnight at $4^{\circ} \mathrm{C}$, followed by incubation with horseradish peroxidase-conjugated secondary goat anti-rabbit antibody (1:200 dilution; PV9001; Zhongshan Biological Technology, Beijing, China) for $30 \mathrm{~min}$ at room temperature. Signals were amplified with use of an ABC kit (Vector Laboratories, Inc., Burlingame, CA, USA) with diaminobenzidine as a chromogen and analyzed by use of Image-Pro Plus 6.0 (Media Cybernetics, Inc., Rockville, MD, USA).

Reverse transcription-quantitative polymerase chain reaction $(R T-q P C R)$. Myocardial tissues were homogenized in TRIzol reagent (Invitrogen; Thermo Fisher Scientific, Inc.) to extract total RNA. Purified RNA $(2 \mu \mathrm{g})$ was treated with DNase and reverse transcribed (RevertAid M-MulV Reverse Transcriptase; Thermo Fisher Scientific, Inc.) following a program of $42^{\circ} \mathrm{C}$ for $1 \mathrm{~h}, 70^{\circ} \mathrm{C}$ for $5 \mathrm{~min}$. qPCR was performed using the following primers: HMGB1 forward, 5'-ACC TGC CGG GAG GAG CAC AA-3' and reverse, 5'-GCC TCT TGG GGG CAT TGG-3'; GAPDH forward, 5'-AGG TCG GTG TGA ACG GAT TTG GG-3' and reverse, 5'-TGT AGA CCA TGT AGT TGA GGT CA-3'. PCR was performed using a thermocycler (IQ5 Real-Time PCR cycler; Bio-Rad Laboratories, Inc., Hercules, CA, USA) with SsoFast EvaGreen Supermix (Bio-Rad Laboratories, Inc.). The program was $30 \mathrm{sec}$ at $95^{\circ} \mathrm{C}$, then 40 cycles of $96^{\circ} \mathrm{C}$ for $5 \mathrm{sec}$ and $56^{\circ} \mathrm{C}$ for $10 \mathrm{sec}$. The mRNA levels of the target genes were normalized to the expression level of GADPH. Relative mean fold change in expression was calculated using the $2^{-\Delta \Delta C q}$ method (26).

TUNEL assay. A TUNEL assay was performed to assess apoptosis. The formalin-fixed paraffin-embedded cardiac tissues were cut into 4- $\mu \mathrm{m}$ sections. Sections were deparaffinized by washing in a graded ethanol series. DNA fragments were determined using an ApopTag in situ apoptosis detection 
Table I. Blood glucose, body weight, heart rate and blood pressure measurements.

\begin{tabular}{|c|c|c|c|}
\hline Parameter & 0 Weeks & 8 Weeks & 12 Weeks \\
\hline \multicolumn{4}{|c|}{ Blood glucose (mmol/l) } \\
\hline Control & $6.88 \pm 1.16$ & $6.35 \pm 1.19$ & $6.11 \pm 1.06$ \\
\hline DM & $6.70 \pm 1.11$ & $22.32 \pm 3.82^{\mathrm{a}}$ & $24.36 \pm 3.37^{\mathrm{a}}$ \\
\hline $\mathrm{DM}+$ ulinastatin & $6.46 \pm 0.94$ & $23.30 \pm 4.19^{\mathrm{a}}$ & $25.23 \pm 3.34^{\mathrm{a}}$ \\
\hline \multicolumn{4}{|l|}{ Body weight (g) } \\
\hline Control & $25.20 \pm 0.66$ & $26.60 \pm 1.13$ & $27.87 \pm 1.72$ \\
\hline DM & $24.99 \pm 0.94$ & $23.48 \pm 1.15^{\mathrm{a}}$ & $24.70 \pm 1.08^{\mathrm{a}}$ \\
\hline $\mathrm{DM}+$ ulinastatin & $25.51 \pm 0.68$ & $23.83 \pm 1.62^{\mathrm{a}}$ & $24.17 \pm 1.65^{\mathrm{a}}$ \\
\hline \multicolumn{4}{|l|}{ Heart rate (bpm) } \\
\hline Control & $606.13 \pm 25.50$ & $607.25 \pm 17.32$ & $610.63 \pm 16.43$ \\
\hline DM & $611.00 \pm 19.65$ & $605.38 \pm 22.13$ & $600.00 \pm 16.75$ \\
\hline $\mathrm{DM}+$ ulinastatin & $615.25 \pm 17.70$ & $598.13 \pm 20.80$ & $603.84 \pm 17.46$ \\
\hline \multicolumn{4}{|c|}{ Systolic blood pressure (mmHg) } \\
\hline Control & $102.15 \pm 10.70$ & $113.00 \pm 13.18$ & $108.24 \pm 13.80$ \\
\hline DM & $108.25 \pm 15.26$ & $106.12 \pm 13.54$ & $102.75 \pm 14.30$ \\
\hline $\mathrm{DM}+$ ulinastatin & $99.13 \pm 12.41$ & $106.38 \pm 12.97$ & $105.00 \pm 12.77$ \\
\hline
\end{tabular}

DM, diabetes mellitus. ${ }^{\text {a }}<0.05$ vs. control group.

kit (ApopTag Plus Peroxidase; Chemicon; EMD Millipore, Billerica, MA, USA), according to the manufacturer's protocol. To quantify apoptosis, the stained sections were examined under an optical microscope (magnification, $\mathrm{x} 400$ ) and the number of TUNEL positive cells was counted in 6 random microscopic fields and quantified by Image-Pro Plus 6.0 (Media Cybernetics, Inc., Rockville, MD, USA).

Western blot analysis. Mouse heart tissues were lysed in radioimmunoprecipitation assay lysis buffer and the extracted protein content was quantified using the bicinchoninic acid method. A total of $20 \mu \mathrm{g}$ protein per lane was separated using $12 \%$ SDS-PAGE and transferred to a nitrocellulose membrane. The membrane was blocked with $5 \%$ nonfat milk for $1 \mathrm{~h}$ at room temperature and subsequently incubated overnight at $4^{\circ} \mathrm{C}$ with the following primary rabbit antibodies: Anti- $\beta$-actin $(1: 1,000$ dilution; \#4970; Cell Signaling Technology, Inc., Danvers, MA, USA), anti-TNF- $\alpha$ (1:500 dilution; ab6671), anti-IL-6 (1:1,000 dilution; ab6672) (both from Abcam), cleaved caspase-3 (1:500 dilution; \#9662), anti-B-cell lymphoma/1-2 (Bcl-2; 1:1,000 dilution; \#3498), anti-Bcl-2-associated X (Bax; 1:1,000 dilution; \#2772), anti-c-Jun N-terminal kinase (JNK; 1:1,000 dilution; \#9252), anti-phospho-JNK (1:1,000 dilution; \#4668), anti-p38 MAPK (1:1,000 dilution; \#9212) and anti-phospho-p38 MAPK (1:1,000 dilution; \#9211) (all from Cell Signaling Technology, Inc.). Membranes were subsequently incubated with a horseradish peroxidase-conjugated secondary antibody $(1: 2,000$ dilution; ZB2301; Zhongshan Biological Technology) for $1 \mathrm{~h}$ at room temperature. Membranes were visualized using an enhanced chemiluminescence kit (EMD Millipore). TNF- $\alpha$, IL-6, cleaved caspase-3, Bcl-2, Bax, JNK, phospho-JNK, p38 MAPK and phosphor-p38 MAPK expression was normalized against $\beta$-actin expression. The bands were developed with an enhanced chemiluminescence kit (EMD Millipore) and quantified by densitometric analysis (Quantity One version 4.64; Bio-Rad Laboratories, Inc.).

ELISA. Cardiac TNF- $\alpha$ (H052; Jiancheng Bioengineering Institute, Nanjing, China) and IL-6 (555240; BD Biosciences, Franklin Lakes, NJ, USA) were assessed using ELISA kits, according to the manufacturer's protocol.

Statistical analysis. Data are presented as the mean \pm standard deviation. Statistical analysis was performed using SPSS version 18.0 (SPSS, Inc., Chicago, IL, USA). The differences were determined by one-way analysis of variance followed by Fisher's least significant difference test. $\mathrm{P}<0.05$ was considered to indicate a statistically significant difference.

\section{Results}

Mouse characteristics. Body weight, plasma glucose levels, heart rate and systolic blood pressure were measured at 0 , 8 , and 12 weeks after the STZ or saline treatment. At 8 and 12 weeks, mice in the DM group had significantly higher RBG and significantly lower body weights compared with the control $(\mathrm{P}<0.05$; Table I). Ulinastatin had no significant effect on blood glucose, body weight, heart rate or systolic blood pressure $(\mathrm{P}>0.05$; Table I).

Ulinastatin ameliorates DM-induced cardiac dysfunction. Echocardiography was used to assess the cardiac function at 0 , 8 and 12 weeks after the STZ injections (Fig. 1). At the baseline, no significant difference was observed in LVEF, E/A ratio and LVIDs among the groups. At 8 weeks, the ratio of early to late mitral inflow velocity (E/A) was significantly reduced in mice 
A

Control

DM

$\mathrm{DM}+$ ulinastatin

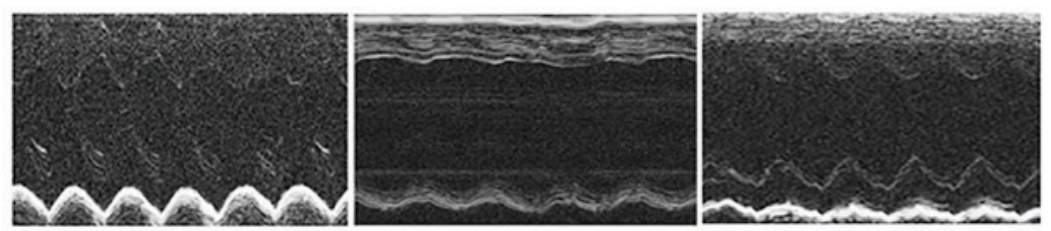

B

C
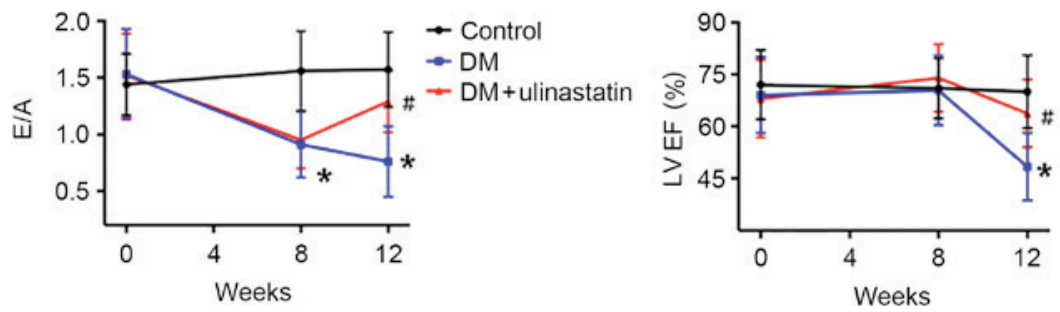

D

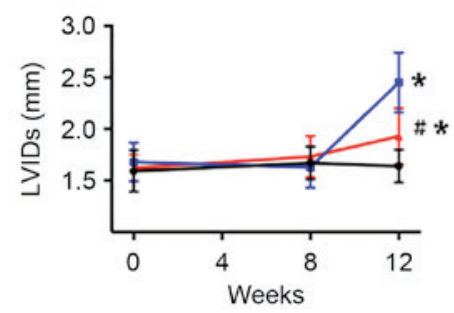

Figure 1. Effect of ulinastatin on heart function in mice with DM. (A) Representative M-mode echocardiograms. (B) E/A, (C) LVEF and (D) LVIDs of the mice. ${ }^{*} \mathrm{P}<0.05$ vs. control and ${ }^{\#} \mathrm{P}<0.05$ vs. DM. E/A, ratio of early to late mitral flow; LVEF, left ventricular ejection fraction; LVIDs, left ventricular internal dimensions in systole; DM, diabetes mellitus.

with DM compared with controls $(\mathrm{P}<0.05$; Fig. 1B). No significant difference was observed in LVEF and LVIDs between the control and DM groups at 8 weeks (Fig. 1C and D). At 12 weeks, E/A remained significantly reduced in mice with DM $(\mathrm{P}<0.05$; Fig. 1B), and these mice displayed echocardiographic evidence of systolic dysfunction and cardiac remodeling compared with controls (Fig. 1A). LVEF was significantly lower $(\mathrm{P}<0.05$; Fig. 1C) and LVIDs were significantly higher $(\mathrm{P}<0.05$; Fig. 1D) in mice with DM compared with controls. Ulinastatin treatment significantly ameliorated the attenuated E/A $(\mathrm{P}<0.05$; Fig. 1B) and LVEF $(\mathrm{P}<0.05$; Fig. 1C) induced by $\mathrm{DM}$, and significantly reduced LVIDs $(\mathrm{P}<0.05$; Fig. 1D) in mice with DM at 12 weeks. These results suggest that mice with DM exhibited diastolic dysfunction after 8 weeks and systolic dysfunction and cardiac remodeling after 12 weeks, and these effects were ameliorated by ulinastatin treatment.

Ulinastatin decreases the levels of HMGB1, TNF- $\alpha$ and IL-6 in diabetic myocardial tissue. Previous results have demonstrated that inflammatory cytokines serve an important role in the progression of DCM (27). To analyze the effect of ulinastatin on the inflammatory response induced by DM, the levels of HMGB1, TNF- $\alpha$ and IL-6 in mice myocardia were examined. We previously reported that HMGB1 serves an important role in DM-induced myocardial fibrosis and dysfunction (15). The protein and mRNA expression levels of HMGB1 in the myocardium were determined by immunohistochemistry (Fig. 2A and B) and RT-qPCR (Fig. 2C), respectively. The results indicate that HMGB1 protein and mRNA levels were significantly increased in mice with DM compared with the control group $(\mathrm{P}<0.05$; Fig. $2 \mathrm{~B}$ and $\mathrm{C})$. However, ulinastatin treatment significantly reduced the DM-induced upregulation of HMGB1 (P<0.05; Fig. 2B and C). Furthermore, western blotting and ELISA results revealed that the levels of TNF- $\alpha$ and IL-6 were significantly increased in mice with DM compared with control mice $(\mathrm{P}<0.05$; Fig. 3). With ulinastatin treatment, the expression of TNF- $\alpha$ and IL- 6 significantly decreased in the diabetic myocardium ( $\mathrm{P}<0.05$; Fig. 3 ). These data suggest that ulinastatin may effectively alleviate the inflammatory response in the diabetic myocardium via the downregulation of HMGB1, TNF- $\alpha$ and IL-6.

Ulinastatin reduces cardiomyocyte apoptosis in mice with DM. TUNEL staining was performed to investigate the effect of ulinastatin on DM-induced cardiomyocyte apoptosis. The number of TUNEL-positive cells in the heart tissue was significantly increased in the DM group compared with the control group ( $\mathrm{P}<0.05$; Fig. 4A), and ulinastatin intervention significantly decreased the number of TUNEL-positive cells compared with that in the untreated DM group $(\mathrm{P}<0.05$; Fig. 4A). The expression of cleaved caspase- 3 and the $\mathrm{Bax} / \mathrm{Bcl}-2$ ratio were significantly elevated in the diabetic myocardium compared with the control $(\mathrm{P}<0.05$; Fig. $4 \mathrm{~B}$ and $\mathrm{C})$. Ulinastatin treatment was demonstrated to significantly ameliorate DM-induced caspase-3 cleavage and significantly reduce the $\mathrm{Bax} / \mathrm{Bcl}-2$ 
A

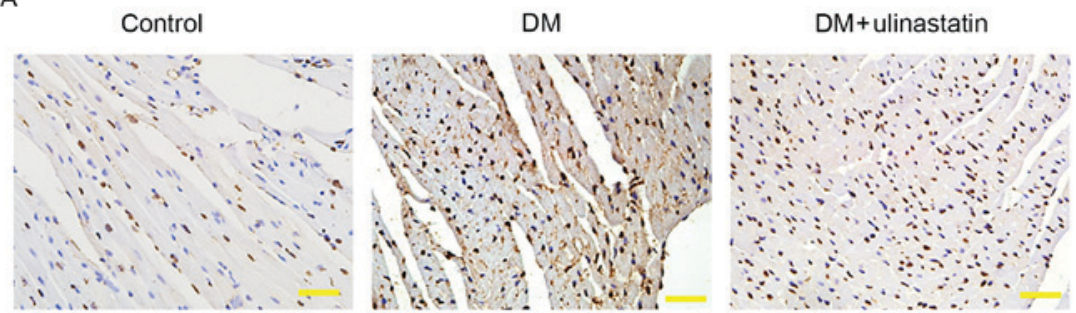

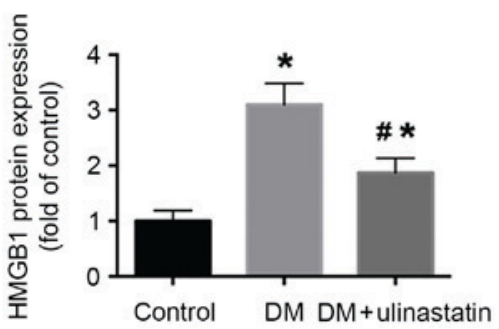

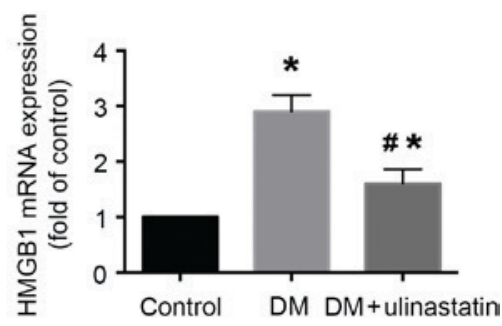

Figure 2. Effect of ulinastatin on HMGB1 levels in mouse myocardia. (A) Immunohistochemical analysis of HMGB1 protein expression in mice myocardia. Myocardial tissues were observed under a light microscope (scale bar, $20 \mu \mathrm{m}$ ). (B) Semi-quantification of HMGB1 staining. (C) Reverse transcription-quantitative polymerase chain reaction analysis of HMGB1 mRNA expression. " $\mathrm{P}<0.05$ vs. control and ${ }^{\#} \mathrm{P}<0.05 \mathrm{vs}$. DM. HMGB1, high mobility group box 1 ; DM, diabetes mellitus.

A

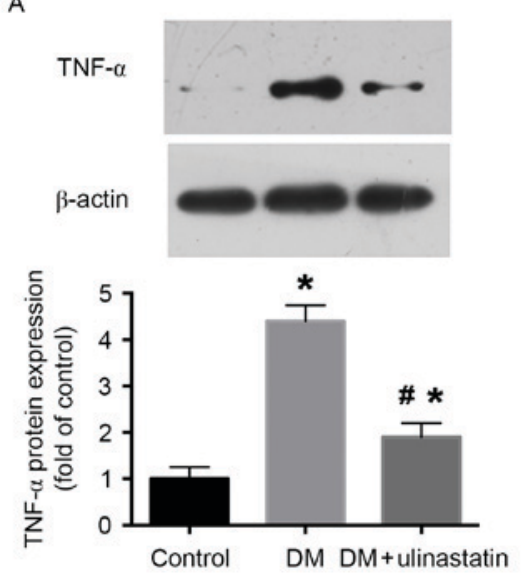

C

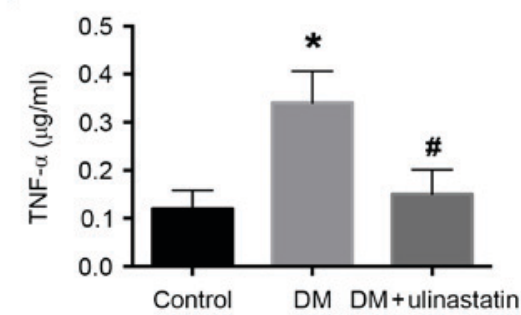

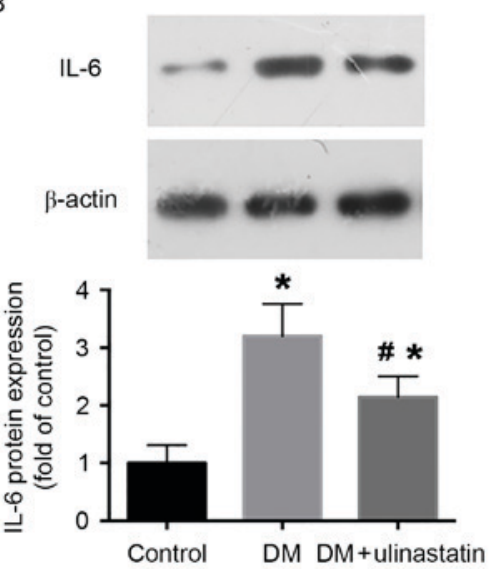

D

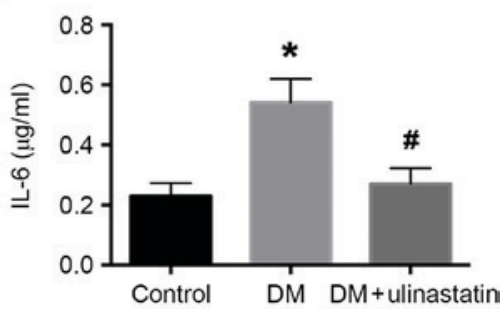

Figure 3. Ulinastatin treatment reduces the expression of TNF- $\alpha$ and IL-6 in mouse myocardia. Western blot analysis of (A) TNF- $\alpha$ and (B) IL-6 protein levels in myocardial tissues. $\beta$-actin was used as a loading control. Cardiac (C) TNF- $\alpha$ and (D) IL-6 levels measured using ELISA. "P<0.05 vs. control and ${ }^{\#} \mathrm{P}<0.05$ vs. DM. TNF- $\alpha$, tumor necrosis factor- $\alpha$; IL-6, interleukin-6; DM, diabetes mellitus.

ratio $(\mathrm{P}<0.05$; Fig. $4 \mathrm{~B}$ and $\mathrm{C})$. These findings indicate that ulinastatin decreases the rate of cardiomyocyte apoptosis and improves cardiac function in a mouse model of DM.

Ulinastatin treatment reduces DM-induced activation of JNK and p38 signaling. The JNK and p38 signaling pathway is a critical pathway that regulates cardiomyocyte apoptosis (28). The effect of ulinastatin on the phosphorylation levels of JNK and p38 induced by DM was assessed, and western blotting results revealed that ulinastatin significantly inhibited the DM-induced increase of JNK $(\mathrm{P}<0.05$; Fig. 5A) and $\mathrm{p} 38$ $(\mathrm{P}<0.05$; Fig. 5B) phosphorylation. 
A Control DM

$\mathrm{DM}+$ ulinastatin
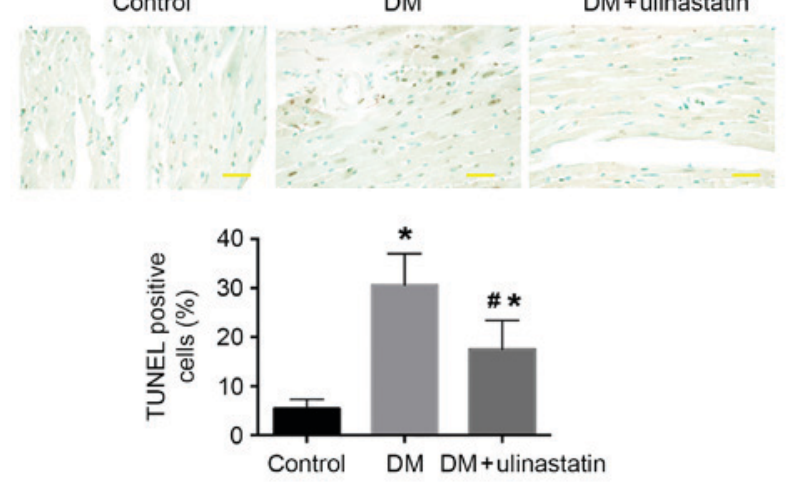

B

C
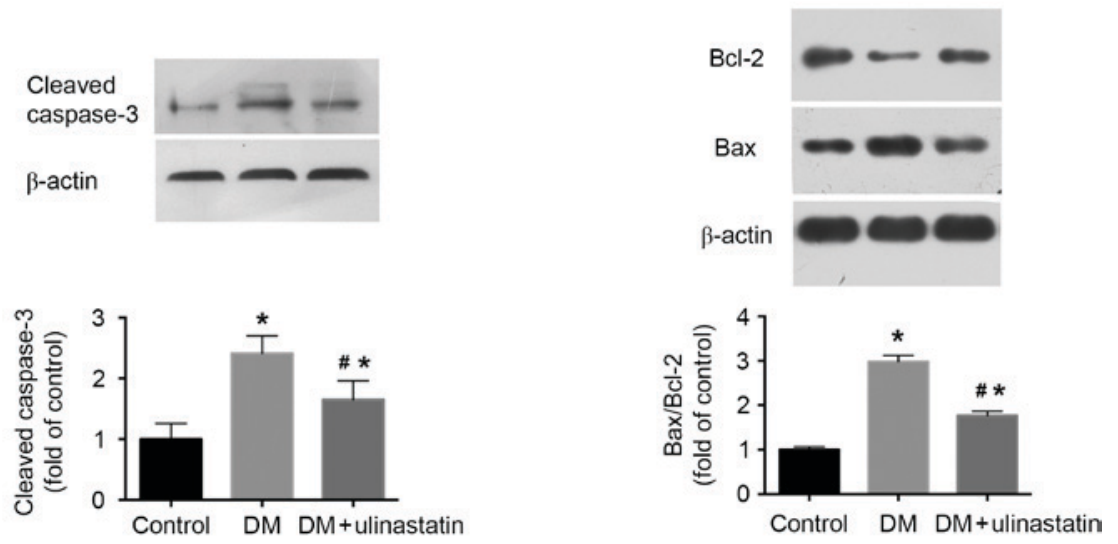

Figure 4. Ulinastatin treatment protects against DM-induced myocardial apoptosis in mice. (A) Cardiomyocyte apoptosis was assessed using TUNEL staining. Apoptotic cells were those with brown staining in the nucleus (scale bar, $20 \mu \mathrm{m}$ ). Western blot analysis of protein levels of (B) cleaved caspase-3, and (C) Bcl-2 and Bax. " $\mathrm{P}<0.05$ vs. control and ${ }^{\#} \mathrm{P}<0.05$ vs. DM. Bcl-2, B-cell lymphoma 2; Bax, Bcl-2-associated X; DM, diabetes mellitus.

A

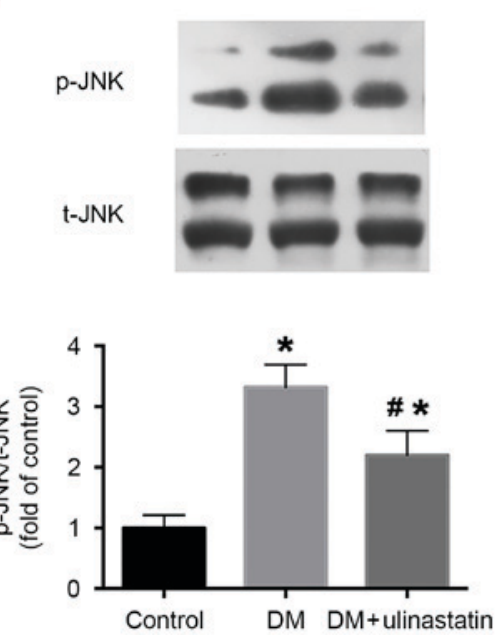

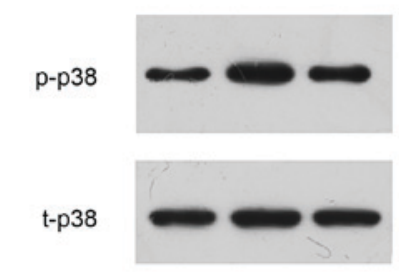

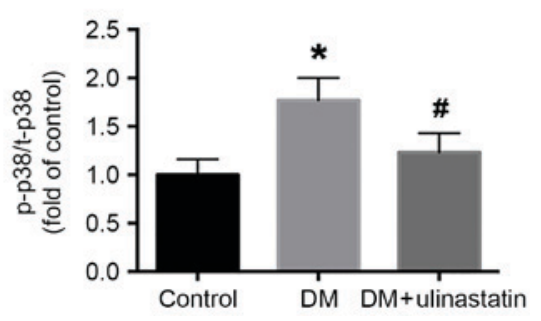

Figure 5. Ulinastatin treatment inhibited DM-induced phosphorylation of JNK and p38 in mice. Western blot analysis of the expression of (A) t-JNK and p-JNK, and (B) t-p38 and p-p38 in mice. " $\mathrm{P}<0.05$ vs. control and ${ }^{\#} \mathrm{P}<0.05$ vs. DM. JNK, c-Jun N-terminal kinase; t, total; p, phosphorylated; DM, diabetes mellitus.

\section{Discussion}

In the present study, the results indicated that ulinastatin improved DM-induced cardiac dysfunction by suppressing inflammatory responses and reducing cardiomyocyte apoptosis, in part via inhibition of the MAPK signaling pathway. This indicated that the administration of ulinastatin has a protective effect in the pathophysiology of DCM.

A previous study reported higher levels of ulinastatin in patients with type 1 and type 2 DM compared with healthy 
controls (29). The increased ulinastatin levels observed in patients with DM may reflect a systemic inflammatory state triggered by hyperglycemia, representing a marker of chronic inflammation in DM. Ulinastatin is a 143-amino acid acidic glycoprotein secreted by the liver and derived from human urine (30). Ulinastatin has been reported to have a myocardial protective effect in various I/R injury models due to its anti-inflammatory properties (31-33). Previous research revealed that ulinastatin is able to improve cardiac function in rabbits following cardiac arrest by inhibiting inflammatory responses and oxidative stress (34). It has been reported that hyperglycemia induces the activation of oxidative stress and inflammatory cascade pathways (35). In the present study, ulinastatin was demonstrated to have a myocardial protective effect in DCM by inhibiting inflammation and apoptosis.

The importance of inflammation in pathogenesis of DCM is well recognized. HMGB1 is a cytokine that is upregulated in the inflammatory process (36). Extracellular HMGB1 is reported to function as an inflammatory cytokine, which is able to initiate and maintain the cascade amplification of inflammation (37). We previously demonstrated that hyperglycemia increased the expression and active secretion of HMGB1 in myocardial cells, and that inhibiting HMGB1 expression may attenuate DM-induced cardiac dysfunction (15). In the present study, HMGB1 expression was decreased in the ulinastatin treatment group compared with the untreated DM group. The results of a previous animal model of DM supported the notion that TNF- $\alpha$ and IL-6 serve a significant role in the inflammation response of DCM (23). The protective effect of ulinastatin may be associated with its anti-inflammatory ability. Previous research reported that ulinastatin was able to suppress the inflammatory response in mice with sepsis (38). Furthermore, ulinastatin has been reported to reduce the expression of TNF- $\alpha$ and IL- 6 and increase levels of IL-10 and IL-13 in lung I/R injury $(20,39)$. The results of the present study demonstrate that the expression levels of TNF- $\alpha$ and IL- 6 were increased in the diabetic myocardium of the mice and this increase was significantly attenuated in ulinastatin-treated mice. These observations indicate that ulinastatin may protect the myocardium against DM-induced injury by inhibiting the synthesis and release of HMGB1, and downregulating TNF- $\alpha$ and IL-6.

Apoptosis of cardiomyocytes is increased in the diabetic heart in patients and animal models, and it has been indicated to be significant in the pathogenesis of DCM (40). Myocardial apoptosis results in a loss of contractile tissue, which initiates cardiac fibrosis and remodeling (41). Bcl-2 and Bax are associated with the regulation of cell apoptosis (42); an increased ratio of $\mathrm{Bax} / \mathrm{Bcl}-2$ leads to the formation of pores in the mitochondria, release of cytochrome $c$, and activation of the apoptotic pathway (43). A previous study reported that ulinastatin treatment ameliorated renal dysfunction via the upregulation of Bcl-2 (13). In the present study, TUNEL staining indicated that ulinastatin treatment significantly reduced cardiomyocyte apoptosis in the diabetic myocardium. Furthermore, it was demonstrated that ulinastatin treatment reduced the DM-induced upregulation in caspase-3 activity and Bax/Bcl-2 ratio in heart. Therefore, in addition to its anti-inflammatory effect, it appears likely that the anti-apoptosis effect of ulinastatin also contributes to its protective function in the mouse model of DM.
It has previously been reported that MAPKs including JNK and p-38 serve important roles in cell death and the production of cytokines (44). DM-induced heart tissue injury is associated with the activation of p38 and JNK (45). Ulinastatin also inhibits the production of TNF- $\alpha$, IL-1 and IL-6, possibly via suppressing the MAPK signaling pathway (46). These pathways comprise important molecular mechanisms responsible for the inflammatory response and the activity of apoptotic kinases (47); therefore, inhibition of JNK and p38 activation may suppress the inflammatory response and limit injury. The results of the present study suggest that ulinastatin is able to reduce the phosphorylation of JNK and p38 in the diabetic heart.

In conclusion, the present study demonstrates that ulinastatin has a protective role against DCM, which is achieved by the suppression of inflammation and cardiomyocyte apoptosis via the JNK and p38 signaling pathway. These results may provide a clinical basis for the use of ulinastatin as a novel therapy for the treatment of DCM.

\section{Acknowledgements}

The present study was supported by the Seed Fund of the Second Hospital of Shandong University (grant no. S2014010003), the Science and Technology Development Plan of Shandong Province (grant no. 2015GSF118078) and the National Natural Science Foundation of China (grant nos. 81500217 and 81502050).

\section{References}

1. Chavali V, Tyagi SC and Mishra PK: Predictors and prevention of diabetic cardiomyopathy. Diabetes Metab Syndr Obes 6: 151-160, 2013.

2. Huynh K, Bernardo BC, McMullen JR and Ritchie RH: Diabetic cardiomyopathy: Mechanisms and new treatment strategies targeting antioxidant signaling pathways. Pharmacol Ther 142: 375-415, 2014.

3. Yu XY, Chen HM, Liang JL, Lin QX, Tan HH, Fu YH, Liu XY, Shan ZX, Li XH, Yang HZ, et al: Hyperglycemic myocardial damage is mediated by proinflammatory cytokine: Macrophage migration inhibitory factor. PLoS One 6: e16239, 2011.

4. Cai L, Li W, Wang G, Guo L, Jiang Y and Kang YJ: Hyperglycemia-induced apoptosis in mouse myocardium: Mitochondrial cytochrome C-mediated caspase-3 activation pathway. Diabetes 51: 1938-1948, 2002.

5. Baraka A and AbdelGawad H: Targeting apoptosis in the heart of streptozotocin-induced diabetic rats. J Cardiovasc Pharmacol Ther 15: 175-181, 2010.

6. Jönsson-Berling BM, Ohlsson $\mathrm{K}$ and Rosengren $\mathrm{M}$ : Radioimmunological quantitation of the urinary trypsin inhibitor in normal blood and urine. Biol Chem Hoppe Seyler 370: 1157-1161, 1989.

7. Nishiyama T, Aibiki M and Hanaoka K: The effect of ulinastatin, a human protease inhibitor, on the transfusion-induced increase of plasma polymorphonuclear granulocyte elastase. Anesth Analg 82: 108-112, 1996

8. Xiao J, Zhu X, Ji G, Yang Q, Kang B, Zhao J, Yao F, Wu L, Ni X and Wang Z: Ulinastatin protects cardiomyocytes against ischemia-reperfusion injury by regulating autophagy through mTOR activation. Mol Med Rep 10: 1949-1953, 2014.

9. Shin IW, Jang IS, Lee SM, Park KE, Ok SH, Sohn JT, Lee HK and Chung YK: Myocardial protective effect by ulinastatin via an anti-inflammatory response after regional ischemia/reperfusion injury in an in vivo rat heart model. Korean J Anesthesiol 61: 499-505, 2011.

10. Shibata T, Yamamoto F, Suehiro S and Kinoshita H: Effects of protease inhibitors on postischemic recovery of the heart. Cardiovasc Drugs Ther 11: 547-556, 1997. 
11. Masuda T, Sato K, Noda C, Ikeda KM, Matsunaga A, Ogura MN, Shimizu K, Nagasawa H, Matsuyama N and Izumi T: Protective effect of urinary trypsin inhibitor on myocardial mitochondria during hemorrhagic shock and reperfusion. Crit Care Med 31: 1987-1992, 2003.

12. Koga Y, Fujita M, Tsuruta R, Koda Y, Nakahara T, Yagi T, Aoki T, Kobayashi C, Izumi T, Kasaoka S, et al: Urinary trypsin inhibitor suppresses excessive superoxide anion radical generation in blood, oxidative stress, early inflammation, and endothelial injury in forebrain ischemia/reperfusion rats. Neurol Res 32: 925-932, 2010.

13. Chen CC, Liu ZM, Wang HH, He W, Wang Y and Wu WD: Effects of ulinastatin on renal ischemia-reperfusion injury in rats. Acta Pharmacol Sin 25: 1334-1340, 2004.

14. Westermann D, Van Linthout S, Dhayat S, Dhayat N, Schmidt A, Noutsias M, Song XY, Spillmann F, Riad A, Schultheiss HP and Tschöpe C: Tumor necrosis factor-alpha antagonism protects from myocardial inflammation and fibrosis in experimental diabetic cardiomyopathy. Basic Res Cardiol 102: 500-507, 2007.

15. Wang WK, Wang B, Lu QH, Zhang W, Qin WD, Liu XJ, Liu XQ, An FS, Zhang Y and Zhang MX: Inhibition of high-mobility group box 1 improves myocardial fibrosis and dysfunction in diabetic cardiomyopathy. Int J Cardiol 172: 202-212, 2014.

16. Tong Y, Tang Z, Yang T, Yang Y, Yang L, Shen W and Chen W: Ulinastatin preconditioning attenuates inflammatory reaction of hepatic ischemia reperfusion injury in rats via high mobility group box 1 (HMGB1) inhibition. Int J Med Sci 11: 337-343, 2014.

17. Mann DL: Inflammatory mediators and the failing heart: Past, present, and the foreseeable future. Circ Res 91: 988-998, 2002.

18. Kocabas E, Sarikçioğlu A, Aksaray N, Seydaoğlu G, Seyhun Y and Yaman A: Role of procalcitonin, C-reactive protein, interleukin-6, interleukin-8 and tumor necrosis factor-alpha in the diagnosis of neonatal sepsis. Turk J Pediatr 49: 7-20, 2007.

19. Bae HB, Jeong CW, Li M, Kim HS and Kwak SH: Effects of urinary trypsin inhibitor on lipopolysaccharide-induced acute lung injury in rabbits. Inflammation 35: 176-182, 2012.

20. Cao YZ, Tu YY, Chen X, Wang BL, Zhong YX and Liu MH: Protective effect of Ulinastatin against murine models of sepsis: Inhibition of TNF- $\alpha$ and IL- 6 and augmentation of IL-10 and IL-13. Exp Toxicol Pathol 64: 543-547, 2012.

21. Matsuzaki H, Kobayashi H, Yagyu T, Wakahara K, Kondo T, Kurita N, Sekino H, Inagaki K, Suzuki M, Kanayama N and Terao T: Bikunin inhibits lipopolysaccharide-induced tumor necrosis factor alpha induction in macrophages. Clin Diagn Lab Immunol 11: 1140-1147, 2004.

22. Kim SJ, Yoo KY, Jeong CW, Kim WM, Lee HK, Bae HB, Kwak $\mathrm{SH}, \mathrm{Li} \mathrm{M}$ and Lee J: Urinary trypsin inhibitors afford cardioprotective effects through activation of PI3K-Akt and ERK signal transduction and inhibition of p38 MAPK and JNK Cardiology 114: 264-270, 2009.

23. Ti Y, Xie GL, Wang ZH, Bi XL, Ding WY, Wang J, Jiang GH, Bu PL, Zhang Y, Zhong $M$ and Zhang W: TRB3 gene silencing alleviates diabetic cardiomyopathy in a type 2 diabetic rat model. Diabetes 60: 2963-2974, 2011.

24. Katoh H, Ishikawa H, Hasegawa M, Yoshida Y, Suzuki Y, Ohno T, Takahashi T and Nakano T: Protective effect of urinary trypsin inhibitor on the development of radiation-induced lung fibrosis in mice. J Radiat Res 51: 325-332, 2010.

25. Feng M, Shu Y, Yang Y, Zheng X, Li R, Wang Y, Dai Y, Qiu W, $\mathrm{Lu} \mathrm{Z}$ and $\mathrm{Hu} \mathrm{X}$ : Ulinastatin attenuates experimental autoimmune encephalomyelitis by enhancing anti-inflammatory responses. Neurochem Int 64: 64-72, 2014.

26. Livak KJ and Schmittgen TD: Analysis of relative gene expression data using real-time quantitative PCR and the 2(-Delta Delta C(T)) method. Methods 25: 402-408, 2001.

27. Palomer X, Salvadó L, Barroso E and Vázquez-Carrera M: An overview of the crosstalk between inflammatory processes and metabolic dysregulation during diabetic cardiomyopathy. Int J Cardiol 168: 3160-3172, 2013.

28. Johnson GL and Lapadat R: Mitogen-activated protein kinase pathways mediated by ERK, JNK, and p38 protein kinases. Science 298: 1911-1912, 2002.
29. Lepedda AJ, Nieddu G, Rocchiccioli S, Fresu P, De Muro P and Formato M: Development of a method for urine bikunin/urinary trypsin inhibitor (UTI) quantitation and structural characterization: Application to type 1 and type 2 diabetes. Electrophoresis 34 : 3227-3233, 2013.

30. Salier JP, Diarra-Mehrpour M, Sesboue R, Bourguignon J, Benarous R, Ohkubo I, Kurachi S, Kurachi K and Martin JP: Isolation and characterization of cDNAs encoding the heavy chain of human inter-alpha-trypsin inhibitor (I alpha TI) Unambiguous evidence for multipolypeptide chain structure of I alpha TI. Proc Natl Acad Sci USA 84: 8272-8276, 1987.

31. Yano T, Anraku S, Nakayama R and Ushijima K: Neuroprotective effect of urinary trypsin inhibitor against focal cerebral ischemia-reperfusion injury in rats. Anesthesiology 98: 465-473, 2003.

32. Wu YJ, Ling Q, Zhou XH, Wang Y, Xie HY, Yu JR and Zheng SS: Urinary trypsin inhibitor attenuates hepatic ischemia-reperfusion injury by reducing nuclear factor-kappa B activation. Hepatobiliary Pancreat Dis Int 8: 53-58, 2009.

33. Shin IW, Jang IS, Lee SM, Park KE, Ok SH, Sohn JT, Lee HK and Chung YK: Myocardial protective effect by ulinastatin via an anti-inflammatory response after regional ischemia/reperfusion injury in an in vivo rat heart model. Korean J Anesthesiol 61: 499-505, 2011

34. Hu CL, Li H, Xia JM, Li X, Zeng X, Liao XX, Zhan H, Jing XL and Dai G: Ulinastatin improved cardiac dysfunction after cardiac arrest in New Zealand rabbits. Am J Emerg Med 31: 768-774, 2013.

35. Falcão-Pires I and Leite-Moreira AF: Diabetic cardiomyopathy: Understanding the molecular and cellular basis to progress in diagnosis and treatment. Heart Fail Rev 17: 325-344, 2012.

36. Scaffidi P, Misteli T and Bianchi ME: Release of chromatin protein HMGB1 by necrotic cells triggers inflammation. Nature 418: 191-195, 2002.

37. Silva E, Arcaroli J, He Q, Svetkauskaite D, Coldren C, Nick JA, Poch K, Park JS, Banerjee A and Abraham E: HMGB1 and LPS induce distinct patterns of gene expression and activation in neutrophils from patients with sepsis-induced acute lung injury. Intensive Care Med 33: 1829-1839, 2007.

38. Huang N, Wang F, Wang Y, Hou J, Li J and Deng X: Ulinastatin improves survival of septic mice by suppressing inflammatory response and lymphocyte apoptosis. J Surg Res 182: 296-302, 2013.

39. Ren B, Wu H, Zhu J, Li D, Shen Y, Ying R, Dong G and Jing H: Ulinastatin attenuates lung ischemia-reperfusion injury in rats by inhibiting tumor necrosis factor alpha. Transplant Proc 38: 2777-2779, 2006

40. Ho FM, Liu SH, Liau CS, Huang PJ and Lin-Shiau SY: High glucose-induced apoptosis in human endothelial cells is mediated by sequential activations of c-Jun $\mathrm{NH}(2)$-terminal kinase and caspase-3. Circulation 101: 2618-2624, 2000.

41. Engel D, Peshock R, Armstong RC, Sivasubramanian N and Mann DL: Cardiac myocyte apoptosis provokes adverse cardiac remodeling in transgenic mice with targeted TNF overexpression. Am J Physiol Heart Circ Physiol 287: H1303-H1311, 2004.

42. Antonsson B and Martinou JC: The Bcl-2 protein family. Exp Cell Res 256: 50-57, 2000.

43. Green DR and Reed JC: Mitochondria and apoptosis. Science 281: 1309-1312, 1998.

44. Srivastava S, Weitzmann MN, Cenci S, Ross FP, Adler S and Pacifici R: Estrogen decreases TNF gene expression by blocking JNK activity and the resulting production of c-Jun and JunD. J Clin Invest 104: 503-513, 1999.

45. Awazu M, Ishikura K, Hida M and Hoshiya M: Mechanisms of mitogen-activated protein kinase activation in experimental diabetes. J Am Soc Nephrol 10: 738-745, 1999.

46. Inoue $\mathrm{K}$ and Takano H: Urinary trypsin inhibitor as a therapeutic option for endotoxin-related inflammatory disorders. Expert Opin Investig Drugs 19: 513-520, 2010.

47. Cargnello M and Roux PP: Activation and function of the MAPKs and their substrates, the MAPK-activated protein kinases. Microbiol Mol Biol Rev 75: 50-83, 2011. 